\title{
Distributionally Robust Mean-Variance Portfolio Selection with Wasserstein Distances*
}

\author{
Jose Blanchet ${ }^{\dagger}$ Lin Chen ${ }^{\ddagger}$ Xun Yu Zhou ${ }^{\S}$ \\ February 15, 2018
}

\begin{abstract}
We revisit Markowitz's mean-variance portfolio selection model by considering a distributionally robust version, where the region of distributional uncertainty is around the empirical measure and the discrepancy between probability measures is dictated by the so-called Wasserstein distance. We reduce this problem into an empirical variance minimization problem with an additional regularization term. Moreover, we extend recent inference methodology in order to select the size of the distributional uncertainty as well as the associated robust target return rate in a data-driven way.
\end{abstract}

Key Words. Mean-variance portfolio selection, robust model, Wasserstein distance, robust Wasserstein profile inference.

\section{Introduction}

We study data-driven mean-variance portfolio selection with model uncertainty (or ambiguity). The classical Markowitz mean-variance model (Markowitz 1952) is to choose a portfolio weighting vector $\phi \in \mathbb{R}^{d}$ (all the vectors in this paper are, by convention, columns) among $d$ stocks to maximize the risk-adjusted expected return. The precise formulation ist 1

$$
\min _{\phi \in \mathbb{R}^{d}}\left\{\phi^{T} \operatorname{Var}_{P^{*}}(R) \phi: \phi^{T} 1=1, \phi^{T} E_{P^{*}}(R)=\rho\right\},
$$

where $R$ is the $d$-dimensional vector of random returns of the stocks; $P^{*}$ is the probability measure underlying the distribution of $R ; E_{P^{*}}$ and $\operatorname{Var}_{P^{*}}$ are respectively

${ }^{*}$ X. Y. Zhou gratefully acknowledges financial support through a start-up grant at Columbia University and through the FDT Center for Intelligent Asset Management. Blanchet gratefully acknowledges support from the National Science Foundation.

${ }^{\dagger}$ Department of Management Science and Engineering, Stanford University, Stanford, California 94305, USA, jblanche@stanford.edu

${ }_{\ddagger}^{\ddagger}$ Department of Industrial Engineering and Operations Research, Columbia University, New York, New York 10027, USA, 1c3110@columbia.edu

$\S$ Department of Industrial Engineering and Operations Research, Columbia University, New York, New York 10027, USA, xz2574@columbia.edu

${ }^{1}$ There are several mathematically equivalent formulations of the original mean-variance model. 
the expectation and variance under $P^{*}$; and $\rho$ is the targeted expected return of the portfolio.

It is well known that this model has a major drawback. On one hand, its solutions are very sensitive to the underlying parameters, namely the mean and the covariance matrix of the stocks. On the other hand, $E_{P^{*}}$ is unknown in practice; so one has to resort to the empirical versions of the mean and the covariance matrix instead, which are usually significantly deviated from the true ones (especially the mean, due to the notorious "mean blur" problem).

This motivates the development of the "robust" formulation of the Markowitz model which recognizes and tries to account for the impact of the (potentially significant) discrepancies between $P^{*}$ and its empirical version. This idea originates in the robust control approach in control theory (see Peterson, James and Dupuis (2000)). Hansen and Sargent (2008) give a systematic account on applications of robust control to economic models. There is also a rich literature on robustification of portfolio choice. Lobo and Boyd (2000) are among the first to provide a worst-case robust analysis with respect to the second-order moment uncertainty within the Markowitz framework. Pflug and Wozabal (2007) formulate a Markowitz model with distributional robustness based on a Wasserstein distance, a metric measuring the discrepancy between two probability measures which we also apply in this paper. Nevertheless, their formulation involves an additional value-at-risk type of constraint that leads to a much more complex optimization problem. More importantly, their choice of the uncertainty size is exogenous and no guidance for optimally selecting the size is given. Esfahani and Kuhn (2017) provide representations for the worst-case expectations in a Wasserstein-based ambiguity set centered at the empirical measure, and then apply their results to portfolio selection using different risk measures, leading to models different from the Markowitz model. The choice of the uncertainty size is suboptimal because it dramatically deteriorates with the dimension of the underlying portfolio.

Along a line different from full distributional uncertainty, Delage and Ye (2010) construct uncertainty regions only involving means and covariances of the return vector. Wozabal (2012) also considers a robust portfolio model with risk constraints based on expected short-fall, resulting in an optimization problem that requires solving multiple convex problems. Again, these papers do not consider the choice of the size of the uncertainty sets.

Papers that address different optimization techniques (such as interior point methods, conic programming and linear matrix inequalities) in solving robust portfolio selection include Halldorsson and Tutuncu (2000), Costa and Paiva (2001) and Ghaoui, Oks and Oustry (2003). Finally, we mention the works of Goh and Sim (2010) and Wisesmann, Kuhn and Sim (2014) who investigate different forms of distributional ambiguity sets, as well as those of Goh and Sim (2013) and Jiang and Guan (2016) who study distributional robust formulations based on the Kullback-Leibler divergences. It is worth noting that the Kullback-Leibler divergence-based formulation is popular in economics (see Hansen and Sargent (2008)).

In this paper, we are interested in studying a distributionally robust optimization (DRO) formulation of the mean-variance problem, given by

$$
\min _{\phi \in \mathcal{F}_{\delta, \bar{\alpha}}(n)} \max _{P \in \mathcal{U}_{\delta}\left(P_{n}\right)}\left\{\phi^{T} \operatorname{Var}_{P}(R) \phi\right\}
$$


where $P_{n}$ is the empirical probability derived from historical information of the sample size $n, \mathcal{U}_{\delta}\left(P_{n}\right):=\left\{P: D_{c}\left(P, P_{n}\right) \leq \delta\right\}$ is the ambiguity set, $\mathcal{F}_{\delta, \bar{\alpha}}(n)=\left\{\phi: \phi^{T} 1=\right.$ $\left.1, \min _{P \in \mathcal{U}_{\delta}\left(P_{n}\right)}\left[E_{P}\left(\phi^{T} R\right)\right] \geq \bar{\alpha}\right\}$ is the feasible region of portfolios, $E_{P}$ and $\operatorname{Var}_{P}(R)$ denote respectively the mean and the covariance matrix under $P$, and $D_{c}(\cdot, \cdot)$ is a notion of discrepancy between two probability measures based on a suitably defined Wasserstein distance.2

Intuitively, formulation (2) introduces an artificial adversary $P$ (whose problem is that of the inner maximization) as a tool to account for the impact of the model uncertainty around the empirical distribution. There are two key parameters, $\delta$ and $\bar{\alpha}$, in this formulation, and they need to be carefully chosen. The parameter $\delta$ can be interpreted as the power given to the adversary: The larger the value of $\delta$ the more power is given. If $\delta$ is too large relative to the evidence (i.e. the size of $n$ ), then the portfolio selection will tend to be unnecessarily conservative. On the other hand, $\bar{\alpha}$ can be regraded as the lowest acceptable target return given the ambiguity set. Naturally, the choice of $\bar{\alpha}$ should be based on the original target $\rho$ given in (1); but one also needs to take into account the size of the distributional uncertainty, $\delta$. Using $\bar{\alpha}=\rho$ will tend to generate portfolios that are too aggressive; it is more sensible to choose $\bar{\alpha}<\rho$ in a way such that $\rho-\bar{\alpha}$ is naturally informed by $\delta$.

This paper makes two main contributions. First, we show that $(2)$ is equivalent to an (explicitly formulated) non-robust minimization problem in terms of the empirical probability measure in which a proper penalty term or "regularization term" is added to the objective function. This connects to the direct use of regularization in variance minimization techniques widely employed both in the machine learning literature and in practice. Indeed, practitioners who use mean-variance portfolio selection models often introduce regularization penalties, inspired by Lasso, in order to enhance the sparsity leading to fewer stocks in their portfolios. Our use of Wasserstein distance to model distributional uncertainty naturally gives rise to a regularization term, suggesting an alternative, yet theoretical, justification for its use in practice. Our result shows that our robust strategies are able to enhance out-of-sample performance with basically the same level of computational tractability as standard mean-variance selection.

Our second main contribution provides guidance on the choice of the size of the ambiguity set, $\delta$, as well as that of the worst mean return target, $\bar{\alpha}$. This is accomplished by adapting and extending the robust Wasserstein profile inference (RWPI) framework, recently introduced and developed by Blanchet, Kang and Murthy (2016), in a data-driven way that combines optimization principles and basic statistical theory under suitable mixing conditions on historical data.

The rest of the paper is organized as follows: We split Section 2 into three parts, with the assumptions discussed in the first part, followed by the tractability of our distributionally robust optimization formulation (culminating with Theorem 1), and the choice of distributional uncertainty (see Theorem 2 and Section 3.2). Some conclusions and extensions are given in Section 4. The technical proofs of our results are given in various appendices at the end of the paper.

\footnotetext{
${ }^{2}$ Recent work by Blanchet, Kang and Murthy (2016) shows that a similar definition of discrepancy in some other models recovers exactly some well-known machine learning algorithms, such as squareroot Lasso and support vector machines.
} 


\section{Formulation and Main Results}

\subsection{Basic notation and assumptions}

In this subsection, we introduce our assumptions and notation, and review some useful concepts.

The mathematical formulation of our problem is given by (2); but we now need to specify the critical measure $D_{c}(\cdot)$. Let $\mathcal{P}\left(\mathbb{R}^{d} \times \mathbb{R}^{d}\right)$ be the space of Borel probability measures supported on $\mathbb{R}^{d} \times \mathbb{R}^{d}$. A given element $\pi \in \mathcal{P}\left(\mathbb{R}^{d} \times \mathbb{R}^{d}\right)$ is associated to a random vector $(U, V)$, where $U \in \mathbb{R}^{d}$ and $V \in \mathbb{R}^{d}$, in the following way: $\pi_{U}(A)=$ $\pi\left(A \times \mathbb{R}^{d}\right)$ and $\pi_{V}(A)=\pi\left(\mathbb{R}^{d} \times A\right)$ for every Borel set $A \subset \mathbb{R}^{d}$, where $\pi_{U}$ and $\pi_{V}$ are respectively the distributions of $U$ and $\pi_{V}$.

Let us introduce a cost function $c: \mathbb{R}^{d} \times \mathbb{R}^{d} \rightarrow[0, \infty]$, which we shall assume to be lower semicontinuous and such that $c(u, u)=0$ for any $u \in \mathbb{R}^{d}$.

Now, given two probability distribution $P$ and $Q$ supported on $\mathbb{R}^{d}$ and a cost function $c$, define

$$
D_{c}(P, Q):=\inf \left\{E_{\pi}[c(U, W)]: \pi \in \mathcal{P}\left(\mathbb{R}^{d} \times \mathbb{R}^{d}\right), \pi_{U}=P, \pi_{W}=Q\right\},
$$

which can be interpreted as the optimal (minimal) transportation cost (also known as the optimal transport discrepancy or the Wasserstein discrepancy) of moving the mass from $P$ into the mass of $Q$ under a cost $c(x, y)$ per unit of mass transported from $x$ to $y$. If for a given $p>0, c^{1 / p}(\cdot)$ is a metric, then so is $D_{c}^{1 / p}$ (see Villani (2003)). Such a metric $D_{c}^{1 / p}$ is known as a Wasserstein distance of order $p$. Most of the times in this paper, we choose the following cost function

$$
c(u, w)=\|w-u\|_{q}^{2}
$$

where $q \geq 1$ is fixed (which leads to a Wasserstein distance of order 2) $]^{3}$ Finally, we define the ambiguity set $\mathcal{U}_{\delta}\left(P_{n}\right)$ as

$$
\mathcal{U}_{\delta}\left(P_{n}\right)=\left\{P: D_{c}\left(P, P_{n}\right) \leq \delta\right\}
$$

where $P_{n}$ is the empirical probability measure with a sample size $n$, i.e

$$
P_{n}(d r)=\frac{1}{n} \sum_{i=1}^{n} \delta_{R_{i}}(d r)
$$

where $R_{i}(i=1,2, \ldots, n)$ are realizations of $R$ and $\delta_{R_{i}}(\cdot)$ is the indicator function.

\subsection{Computational tractability}

We now reformulate (2) in a way that becomes computationally tractable. The first step is to show that the feasible region over $\phi$ in the outer minimization part can be explicitly evaluated. This is given in the following proposition, whose proof is relegated to the Appendix.

\footnotetext{
${ }^{3}$ Different cost functions can be used, resulting in different regularization penalties, as we will discuss in Section 5: Concluding Remarks.
} 
Proposition 1 For $c(u, w)=\|u-w\|_{q}^{2}, q \geq 1$, we have

$$
\min _{P \in \mathcal{U}_{\delta}\left(P_{n}\right)} E_{P}\left(\phi^{T} R\right)=E_{P_{n}}\left(\phi^{T} R\right)-\sqrt{\delta}\|\phi\|_{p},
$$

with $1 / p+1 / q=1$.

Therefore, the feasible region is equivalent to

$$
\mathcal{F}_{\delta, \bar{\alpha}}(n)=\left\{\phi: \phi^{T} 1=1, E_{P_{n}}\left(\phi^{T} R\right) \geq \bar{\alpha}+\sqrt{\delta}\|\phi\|_{p}\right\},
$$

which can now be seen as clearly convex.

Next, by fixing $E_{P}\left(\phi^{T} R\right)=\alpha \geq \bar{\alpha}$ in the inner maximization portion of problem (2) we obtain the following equivalent reformulation

$$
\min _{\phi \in \mathcal{F}_{\delta, \bar{\alpha}}}\left\{\max _{\alpha \geq \bar{\alpha}}\left[\max _{P \in \mathcal{U}_{\delta}\left(P_{n}\right), E_{P}\left(\phi^{T} R\right)=\alpha}\left\{\phi^{T} E_{P}\left(R R^{T}\right) \phi\right\}-\alpha^{2}\right]\right\} .
$$

Introducing $E_{P}\left(\phi^{T} R\right)=\alpha$ is useful because the inner-most maximization problem is now linear in $P$. So, let us concentrate on the problem

$$
\max _{P \in \mathcal{U}_{\delta}\left(P_{n}\right), E_{P}\left(\phi^{T} R\right)=\alpha} \phi^{T} E_{P}\left[R R^{T}\right] \phi .
$$

The following proposition solves this problem in terms of a general cost function $c$.

Proposition 2 For an arbitrary cost function $c$ that is lower semicontinuous and non-negative, the optimal value function of problem (7) is given by

$$
\inf _{\lambda_{1} \geq 0, \lambda_{2}}\left[\frac{1}{n} \sum_{i=1}^{n} \Phi\left(R_{i}\right)+\lambda_{1} \delta+\lambda_{2} \alpha\right]
$$

where

$$
\Phi\left(R_{i}\right):=\sup _{u}\left[\left(\phi^{T} u\right)^{2}-\lambda_{1} c\left(u, R_{i}\right)-\lambda_{2} \phi^{T} u\right] .
$$

A proof, based on a dual argument, is given in the Appendix. Thanks to this proposition, we are able to reduce the inner (infinite dimensional) variational problem in (2) into a two-dimensional optimization problem in terms of $\lambda_{1}$ and $\lambda_{2}$, which can be further simplified if the cost function $c$ has additional structure. We make this statement precise in the case of a quadratic $l_{q}$ cost.

Proposition 3 Let $c(u, w)=\|u-w\|_{q}^{2}$ with $q \geq 1$ and $1 / p+1 / q=1$. If $(\alpha-$ $\left.\phi^{T} E_{P_{n}}[R]\right)^{2}-\delta\|\phi\|_{p}^{2} \leq 0$, then the value of (7) is equal to

$$
\begin{aligned}
h(\alpha, \phi):= & E_{P_{n}}\left[\left(\phi^{T} R\right)^{2}\right]+2\left(\alpha-\phi^{T} E_{P_{n}}[R]\right) \phi^{T} E_{P_{n}}[R]+\delta\|\phi\|_{p}^{2} \\
& +2 \sqrt{\delta\|\phi\|_{p}^{2}-\left(\alpha-\phi^{T} E_{P_{n}}[R]\right)^{2}} \sqrt{\phi^{T} \operatorname{Var}_{P_{n}}(R) \phi} .
\end{aligned}
$$

Again, a proof of this proposition is in the Appendix. The condition $(\alpha-$ $\left.\phi^{T} E_{P_{n}}[R]\right)^{2}-\delta\|\phi\|_{p}^{2} \leq 0$ is to make sure that (7) is feasible, failing which the optimal value $h(\alpha, \phi)=-\infty$. Proposition 3 ultimately leads to the following main result of the paper, one that transforms (2) into a non-robust portfolio selection problem in terms of the empirical measure $P_{n}$. 
Theorem 1 The primal formulation given in (2) is equivalent to the following dual problem

$$
\min _{\phi \in \mathcal{F}_{\delta, \bar{\alpha}}(n)}\left(\sqrt{\phi^{T} \operatorname{Var}_{P_{n}}(R) \phi}+\sqrt{\delta}\|\phi\|_{p}\right)^{2},
$$

in the sense that the two problems have the same optimal solutions and optimal value.

Proof Note that

$$
\begin{aligned}
& h(\alpha, \phi)-\alpha^{2} \\
& =E_{P_{n}}\left[\left(\phi^{T} R\right)^{2}\right]+2\left(\alpha-\phi^{T} E_{P_{n}}[R]\right) \phi^{T} E_{P_{n}}[R]-\alpha^{2}+\delta\|\phi\|_{p}^{2} \\
& +2 \sqrt{\delta\|\phi\|_{p}^{2}-\left(\alpha-\phi^{T} E_{P_{n}}[R]\right)^{2}} \sqrt{\phi^{T} \operatorname{Var}_{P_{n}}(R) \phi} \\
& =E_{P_{n}}\left[\left(\phi^{T} R\right)^{2}\right]+2 \alpha \phi^{T} E_{P_{n}}[R]-\left(\phi^{T} E_{P_{n}}[R]\right)^{2}-\alpha^{2}-\left(\phi^{T} E_{P_{n}}[R]\right)^{2}+\delta\|\phi\|_{p}^{2} \\
& +2 \sqrt{\delta\|\phi\|_{p}^{2}-\left(\alpha-\phi^{T} E_{P_{n}}[R]\right)^{2}} \sqrt{\phi^{T} \operatorname{Var}_{P_{n}}(R) \phi} \\
& =\phi^{T} \operatorname{Var}_{P_{n}}(R) \phi+\left\{\delta\|\phi\|_{p}^{2}-\left(\alpha-\phi^{T} E_{P_{n}}[R]\right)^{2}\right\} \\
& +2 \sqrt{\delta\|\phi\|_{p}^{2}-\left(\alpha-\phi^{T} E_{P_{n}}[R]\right)^{2}} \sqrt{\phi^{T} \operatorname{Var}_{P_{n}}(R) \phi} \\
& =\left(\sqrt{\phi^{T} \operatorname{Var}_{P_{n}}(R) \phi}+\sqrt{\delta\|\phi\|_{p}^{2}-\left(\alpha-\phi^{T} E_{P_{n}}[R]\right)^{2}}\right)^{2} .
\end{aligned}
$$

Therefore, it follows from Proposition 3 that

$$
\max _{\alpha \geq \bar{\alpha},\left(\alpha-\phi^{T} E_{P_{n}}[R]\right)^{2}-\delta\|\phi\|_{p}^{2} \leq 0}\left[h(\alpha, \phi)-\alpha^{2}\right]=\left(\sqrt{\phi^{T} \operatorname{Var}_{P_{n}}(R) \phi}+\sqrt{\delta}\|\phi\|_{p}\right)^{2},
$$

with the optimal $\alpha_{o p t}=\phi^{T} E_{P_{n}}[R] \geq \bar{\alpha}$. This concludes the result.

Because the mapping $\phi \rightarrow \phi^{T} \operatorname{Var}_{P}(R) \phi$ is convex and the feasible region $\mathcal{F}_{\delta, \bar{\alpha}}(n)$ is convex, (9) and therefore (2) are both convex optimization problems. As such, they are tractable optimization problems. Moreover, our approach justifies the regularization technique in mean-variance portfolio selection often adopted in practical settings.

\section{Choice of Model Parameters}

There are two key parameters, $\delta$ and $\bar{\alpha}$, in the formulation (2), the choice of which is not only curious in theory, but also crucial in practical implementation and for the success of our algorithm. The idea is that the choice of these parameters should be informed by the data (i.e. in a data-driven way) based on some statistical principles, rather than arbitrarily exogenous. Specifically, we define the distributional uncertainty region just large enough so that the correct optimal portfolio (the one which we would apply if the underlying distribution was known) becomes a plausible choice with a sufficiently high confidence level.

We need to impose several statistical assumptions.

A1) The underlying return time series $\left(R_{k}: k \geq 0\right)$ is a stationary, ergodic process satisfying $E_{P^{*}}\left(\left\|R_{k}\right\|_{2}^{4}\right)<\infty$ for each $k \geq 0$. Moreover, for each measurable $g(\cdot)$ such 
that $|g(x)| \leq c\left(1+\|x\|_{2}^{2}\right)$ for some $c>0$, the limit

$$
\Upsilon_{g}:=\lim _{n \rightarrow \infty} \operatorname{Var}_{P^{*}}\left(n^{-1 / 2} \sum_{k=1}^{n} g\left(R_{k}\right)\right)
$$

exists and

$$
n^{1 / 2}\left[E_{P_{n}}(g(R))-E_{P^{*}}(g(R))\right] \Rightarrow N\left(0, \Upsilon_{g}\right),
$$

where (and from that time on) " $\Rightarrow$ " denotes weak convergence.

A2) For any matrix $\Lambda \in \mathbb{R}^{d \times d}$ and any vector $\zeta \in \mathbb{R}^{d}$ such that either $\Lambda \neq 0$ or $\zeta \neq 0$

$$
P^{*}\left(\|\Lambda R+\zeta\|_{2}>0\right)>0 \text {. }
$$

A3) The classical model (1) has a unique solution $\phi^{*}$. Moreover, $\operatorname{Var}_{P^{*}}\left[E_{P^{*}}\left(R^{T}\right) R\right]>$ 0 .

Assumption A1) is standard for most time series models (after removing seasonality). Assumption A2) can be easily checked assuming, for example, that $R$ has a density. Assumption A3) is a technical assumption which can be relaxed, but then the evaluation of the optimal choice of $\delta$ would become more cumbersome, as we shall explain.

\subsection{Choice of $\delta$}

The choice of the uncertainty size $\delta$ is crucial. If $\delta$ is too large, then there is too much model ambiguity and the available data becomes less relevant. In this case the resulting optimal portfolios will tend to be just equal allocations. If $\delta$ is too small, then the effect of robustification will be negligible. Therefore, the choice of $\delta$ should not be exogenously specified; rather it should be endogenously informed by the data.

Theorem 1 actually suggests an appropriate order of $\delta=\delta_{n}$ (here $n$ is the size of the available return time series data) in terms of $n$. Because the differences between the optimal value obtained by solving (1) and that obtained by solving the empirical version of (1) are of order $O\left(n^{-1 / 2}\right)$, it follows from Theorem 1 that any choice of $\delta_{n}$ in the order of $o\left(n^{-1}\right)$ would be too small. Hence, an "optimal" order of $\delta_{n}$ should be $O\left(n^{-1}\right)$.

In order to choose an appropriate $\delta_{n}$, here we follow the idea behind the RWPI approach introduced in Blanchet, Kang and Murthy (2016).

Intuitively, the set $\mathcal{U}_{\delta}\left(P_{n}\right)=\left\{P: D_{c}\left(P, P_{n}\right) \leq \delta\right\}$ contains all the probability measures that are plausible variations of the data represented by $P_{n}$. Denote by $\mathcal{Q}(P)$ the Markowitz portfolio selection problem with target return $\rho$ assuming that $P$ is the underlying model:

$$
\begin{aligned}
& \min _{1^{T} \phi=1} \phi^{T} E_{P}\left[R R^{T}\right] \phi \\
& \text { s.t } \phi^{T} E_{P}[R]=\rho,
\end{aligned}
$$


and by $\phi_{P}$ a solution to $\mathcal{Q}(P)$ and $\Phi_{P}$ the set of all such solutions. According to Assumption A3) we have $\Phi_{P^{*}}=\left\{\phi^{*}\right\}$ for some portfolio $\phi^{*}$. Therefore there exist (unique) Lagrange multipliers $\lambda_{1}^{*}$ and $\lambda_{2}^{*}$ such that

$$
\begin{aligned}
2 E_{P^{*}}\left(R R^{T}\right) \phi^{*}-\lambda_{1}^{*} E_{P^{*}}[R]-\lambda_{2}^{*} 1 & =0, \\
\left(\phi^{*}\right)^{T} E_{P^{*}}[R]-\rho & =0 .
\end{aligned}
$$

Because $P_{n} \Rightarrow P_{*}$ under mild assumptions (e.g. A1)), it is reasonable to estimate $\phi^{*}$ by computing $\phi_{P_{n}}$. Now, when $\delta$ is suitably chosen, since $\mathcal{U}_{\delta}\left(P_{n}\right)$ constitutes the models that are plausible variations of $P_{n}$, any $\phi_{P}$ with $P \in \mathcal{U}_{\delta}\left(P_{n}\right)$ is a plausible estimate of $\phi^{*}$. This intuition motivates the definition of the following set

$$
\Lambda_{\delta}\left(P_{n}\right)=\cup_{P \in \mathcal{U}_{\delta}\left(P_{n}\right)} \Phi_{P}
$$

which corresponds to all the plausible estimates of $\phi^{*}$. As a result, $\Lambda_{\delta}\left(P_{n}\right)$ is a natural confidence region for $\phi^{*}$ and, therefore, $\delta$ should be chosen as the smallest number $\delta_{n}^{*}$ such that $\phi^{*}$ belongs to this region with a given confidence level. Namely,

$$
\delta_{n}^{*}=\min \left\{\delta: P^{*}\left(\phi^{*} \in \Lambda_{\delta}\left(P_{n}\right)\right) \geq 1-\delta_{0}\right\},
$$

where $1-\delta_{0}$ is a user-defined confidence level (typically $95 \%$ ).

However, by the mere definition, it is difficult to compute $\delta_{n}^{*}$. We now provide a simpler representation for $\delta_{n}^{*}$ via an auxiliary function called the robust Wasserstein profile (RWP) function. To this end, first observe that any $\phi \in \Lambda_{\delta}\left(P_{n}\right)$ if and only if there exist $P \in \mathcal{U}_{\delta}\left(P_{n}\right)$ and $\lambda_{1}, \lambda_{2} \in(-\infty, \infty)$ such that

$$
\begin{aligned}
2 E_{P}\left(R R^{T}\right) \phi-\lambda_{1} E_{P}[R]-\lambda_{2} 1 & =0, \\
\phi^{T} E_{P}(R)-\rho & =0 .
\end{aligned}
$$

From these two equations, multiplying the first equation by $\phi$, substituting the expression in the second equation and noting that $\phi \cdot 1=1$, we obtain

$$
\lambda_{2}=2(\phi)^{T} E_{P}\left(R R^{T}\right) \phi-\lambda_{1} \rho .
$$

We now define the following RWP function

$$
\overline{\mathcal{R}}_{n}\left(\phi, \lambda_{1}, \Sigma, \mu\right):=\inf \left\{D_{c}\left(P, P_{n}\right):\left\{\begin{array}{l}
2 \Sigma \phi-\lambda_{1} \mu=\left(2(\phi)^{T} \Sigma \phi-\lambda_{1} \mu \cdot \phi\right) 1 \\
\mu=E_{P}[R], \Sigma=E_{P}\left(R R^{T}\right)
\end{array}\right\}\right.
$$

for $\left(\phi, \lambda_{1}, \Sigma, \mu\right) \in \mathbb{R}^{d} \times \mathbb{R} \times \mathcal{S}_{+}^{d \times d} \times \mathbb{R}^{d}$ where $\mathcal{S}_{+}^{d \times d}$ is the set of all the symmetric positive semidefinite matrices and we convent that inf $\emptyset:=+\infty$. Moreover, we define

$$
\overline{\mathcal{R}}_{n}^{*}\left(\phi^{*}\right):=\min _{\Sigma \in \mathcal{S}_{+}^{d \times d}, \mu \in \mathbb{R}^{d}, \lambda_{1} \in \mathbb{R}} \overline{\mathcal{R}}_{n}\left(\phi^{*}, \lambda_{1}, \Sigma, \mu\right) .
$$

It follows directly from the definitions that

$$
\phi^{*} \in \Lambda_{\delta}\left(P_{n}\right) \Longleftrightarrow \overline{\mathcal{R}}_{n}^{*}\left(\phi^{*}\right) \leq \delta .
$$


Therefore

$$
\delta_{n}^{*}=\inf \left\{\delta: P^{*}\left(\overline{\mathcal{R}}_{n}^{*}\left(\phi^{*}\right) \leq \delta\right) \geq 1-\delta_{0}\right\} .
$$

In other words, $\delta_{n}^{*}$ is the quantile corresponding to the $1-\delta_{0}$ percentile of the distri-

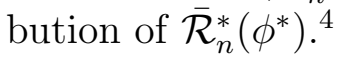

Still, even under A3), the statistic $\overline{\mathcal{R}}_{n}^{*}\left(\phi^{*}\right)$ is somewhat cumbersome to work with as it is derived from solving a minimization problem in terms of the mean and variance. So, instead, we will define an alternative statistic involving only the empirical mean and variance while producing an upper bound which still preserves the target rate of convergence to zero as $n \rightarrow \infty$ (which, as we have argued, should be of order $O\left(n^{-1}\right)$ ).

Denote $\Sigma_{n}=E_{P_{n}}\left(R R^{T}\right)$, and let $\lambda_{1}^{*}$ be the Lagrange multiplier in (11). Set

$$
\mu_{n}=\rho 1+2\left(\Sigma_{n} \phi^{*}-\phi^{* T} \Sigma_{n} \phi^{*} 1\right) / \lambda_{1}^{*} .
$$

Define

$$
\mathcal{R}_{n}\left(\Sigma_{n}, \mu_{n}\right):=\overline{\mathcal{R}}_{n}\left(\phi^{*}, \lambda_{1}^{*}, \Sigma_{n}, \mu_{n}\right)
$$

It is clear that

$$
\mathcal{R}_{n}\left(\Sigma_{n}, \mu_{n}\right) \geq \overline{\mathcal{R}}_{n}^{*}\left(\phi^{*}\right)
$$

Therefore,

$$
\mathcal{R}_{n}\left(\Sigma_{n}, \mu_{n}\right) \leq \delta \Longrightarrow \overline{\mathcal{R}}_{n}^{*}\left(\phi^{*}\right) \leq \delta
$$

and, consequently,

$$
\bar{\delta}_{n}^{*}=\inf \left\{\delta \geq 0: P^{*}\left(\mathcal{R}_{n}\left(\Sigma_{n}, \mu_{n}\right) \leq \delta\right) \geq 1-\delta_{0}\right\} \geq \delta_{n}^{*} .
$$

Moreover, because of the choice of $\Sigma_{n}$ and $\mu_{n}$, we have

$$
\mathcal{R}_{n}\left(\Sigma_{n}, \mu_{n}\right)=\inf \left\{\mathcal{D}_{c}\left(P, P_{n}\right): E_{P}\left[R R^{T}\right]=\Sigma_{n}, E_{P}[R]=\mu_{n}\right\} .
$$

The next result shows $\bar{\delta}_{n}^{*}=O\left(n^{-1}\right)$ as $n \rightarrow \infty$.

Theorem 2 Assume A1) and A2) hold and write $\mu_{*}=E_{P^{*}}(R)$ and $\Sigma_{*}=E_{P^{*}}\left(R R^{T}\right)$. Define

$$
g(x)=x x^{T}+2\left(x \cdot \phi^{*}-\phi^{* T} x \phi^{*} 1\right) / \lambda_{1}^{*} .
$$

Then

$$
n R_{n}\left(\Sigma_{n}, \mu_{n}\right) \Rightarrow L_{0}:=\sup _{\bar{\lambda} \in \mathbb{R}^{d}}\left(\bar{\lambda}^{T} Z-\inf _{\bar{\Lambda} \in \mathbb{R}^{d \times d}} E_{P^{*}}\left[\|\bar{\Lambda} R+\bar{\lambda}\|_{p}^{2}\right]\right)
$$

where $Z \sim N\left(0, \Upsilon_{g}\right)$. Moreover, if $p=2$ then

$$
L_{0}=\|Z\|_{2}^{2} /\left(1-\left\|\mu_{*}\right\|_{2}^{4} / \mu_{*}^{T} \Sigma_{*} \mu_{*}\right) .
$$

A proof of Theorem 2 is provided in the Appendix.

Note that $L_{0}$ has an explicit expression when $p=2$. When $p \neq 2$, using the inequalities that $\|x\|_{p}^{2} \geq\|x\|_{2}^{2}$ if $p<2$ and $d^{\left(\frac{1}{2}-\frac{1}{p}\right)}\|x\|_{p}^{2} \geq\|x\|_{2}^{2}$ if $p>2$, we can find a stochastic upper bound of $L_{0}$ that can be explicitly expressed. In that case we can obtain $\bar{\delta}_{n}^{*}$ in exactly the same way.

\footnotetext{
${ }^{4}$ Herein the analysis is under Assumption A3). If $\Phi_{P^{*}}$ contained more than just one element, then there would be several possible options to formulate an optimization problem for choosing $\delta$. For example, we may choose $\delta$ as the smallest uncertainty size such that $\Phi_{P^{*}} \subset \Lambda_{\delta}\left(P_{n}\right)$ with probability $1-\delta_{0}$, in which case we would need to study $\sup _{\phi^{*} \in \Phi_{P^{*}}} \overline{\mathcal{R}}_{n}^{*}\left(\phi^{*}\right)$.
} 


\subsection{Choice of $\bar{\alpha}$}

Once $\delta$ has been chosen, the next step is to choose $\bar{\alpha}$. The idea is to select $\bar{\alpha}$ just large enough to make sure that we do not rule out that $\phi^{*} \in \mathcal{F}_{\delta, \bar{\alpha}}(n)$ holds with a given confidence level chosen by the user, where $\phi^{*}$ is the optimal solution to (1). It is equivalent to choose $v_{0}$ where

$$
\bar{\alpha}=\rho-\sqrt{\delta}\left\|\phi^{*}\right\|_{p} v_{0}
$$

Therefore, it follows from Proposition 1 that $\phi^{*} \in \mathcal{F}_{\delta, \bar{\alpha}}(n)$ if and only if

$$
\left(\phi^{*}\right)^{T} E_{P_{n}}(R)-\sqrt{\delta}\left\|\phi^{*}\right\|_{p} \geq \rho-\sqrt{\delta}\left\|\phi^{*}\right\|_{p} v_{0} .
$$

However, $\rho=\left(\phi^{*}\right)^{T} E_{P^{*}}(R)$; so the previous inequality holds if and only if

$$
\left(\phi^{*}\right)^{T}\left(E_{P_{n}}(R)-E_{P^{*}}(R)\right) \geq\left\|\phi^{*}\right\|_{p} \sqrt{\delta}\left(1-v_{0}\right) .
$$

Therefore, we can choose $\sqrt{\delta}\left(1-v_{0}\right)<0$ sufficiently negative so that the previous inequality holds with a specified confidence level. We hope to choose a $v_{0}$ such that $\phi^{*}$ will satisfy (13) with confidence level $1-\epsilon$. This can be achieved asymptotically by the central limit theorem as the following result indicates.

Proposition 4 Suppose that A1) and A3) hold and let $\left\{\phi_{n}^{*}\right\}_{n=1}^{\infty}$ be any consistent sequence of estimators of $\phi^{*}$ in the sense that $\phi_{n}^{*} \rightarrow \phi^{*}$ in probability as $n \rightarrow \infty$. Then,

$$
n^{1 / 2}\left[\frac{\left(\phi_{n}^{*}\right)^{T}\left(E_{P_{n}}(R)-E_{P^{*}}(R)\right)}{\left\|\phi_{n}^{*}\right\|_{p}}\right] \Rightarrow N\left(0, \Upsilon_{\phi^{*}}\right),
$$

as $n \rightarrow \infty$, where

$$
\Upsilon_{\phi^{*}}:=\lim _{n \rightarrow \infty} \operatorname{Var}_{P^{*}}\left(n^{-1 / 2} \sum_{k=1}^{n}\left(\phi^{*}\right)^{T} R_{k} /\left\|\phi^{*}\right\|_{p}\right) .
$$

Proof Note that

$$
\begin{aligned}
n^{1 / 2}\left[\frac{\left(\phi_{n}^{*}\right)^{T}\left(E_{P_{n}}(R)-E_{P^{*}}(R)\right)}{\left\|\phi_{n}^{*}\right\|_{p}}\right] & =n^{1 / 2}\left[\frac{\left(\phi_{n}^{*}-\phi^{*}\right)^{T}\left(E_{P_{n}}(R)-E_{P^{*}}(R)\right)}{\left\|\phi_{n}^{*}\right\|_{p}}\right] \\
& +n^{1 / 2}\left[\frac{\left(\phi^{*}\right)^{T}\left(E_{P_{n}}(R)-E_{P^{*}}(R)\right)}{\left\|\phi^{*}\right\|_{p}} \cdot \frac{\left\|\phi^{*}\right\|_{p}}{\left\|\phi_{n}^{*}\right\|_{p}}\right] .
\end{aligned}
$$

By the central limit theorem and the fact that $\phi_{n}^{*} \rightarrow 0$ in probability, we conclude

$$
n^{1 / 2}\left[\frac{\left(\phi_{n}^{*}\right)^{T}\left(E_{P_{n}}(R)-E_{P^{*}}(R)\right)}{\left\|\phi_{n}^{*}\right\|_{p}}-\frac{\left(\phi^{*}\right)^{T}\left(E_{P_{n}}(R)-E_{P^{*}}(R)\right)}{\left\|\phi^{*}\right\|_{p}}\right] \Rightarrow 0
$$

as $n \rightarrow \infty$. However,

$$
n^{1 / 2}\left[\frac{\left(\phi^{*}\right)^{T}\left(E_{P_{n}}(R)-E_{P^{*}}(R)\right)}{\left\|\phi^{*}\right\|_{p}}\right] \Rightarrow N\left(0, \Upsilon_{\phi^{*}}\right)
$$


which implies the desired result.

Using the previous result we can estimate $v_{0}$ asymptotically. Let $\phi_{n}$ denote the optimal solution of problem $\mathcal{Q}\left(P_{n}\right)$. We know that $\phi_{n}$ converges to $\phi^{*}$ in probability. So, in practice we choose a $v_{0}$ such that the following inequality will hold with confidence level $1-\epsilon$,

$$
\frac{1}{\left\|\phi_{n}\right\|_{p}}\left(\phi_{n}\right)^{T}\left(E_{P_{n}}(R)-E_{P^{*}}(R)\right) \geq \sqrt{\delta}\left(1-v_{0}\right) .
$$

The left-hand side of (14) is approximately normally distributed and thus we can choose its $1-\epsilon$ quantile and then we are be able to decide the value of $v_{0}>1$.

\section{Concluding Remarks}

We have provided a data-driven DRO theory for Markowitz's mean-variance portfolio selection. The robust model can be solved via a non-robust one based on the empirical probability measure with an additional regularization term. The size of the distributional uncertainty region is not exogenously given; rather it is informed by the return data in a scheme which we have developed in this paper.

Our results may be generalized in different directions. We have chosen the $l_{q}$ norm in defining our Wasserstein distance due to its popularity in regularization, but other transportation costs can be used. For example, one may consider the type of transportation cost related to adaptive regularization that has been studied by Blanchet, Kang, Zhang and Murthy (2017), or the one related to industry cluster as in Blanchet and Kang (2017). Another significant direction is a dynamic (discretetime or continuous-time) version of the DRO Markowitz model.

\section{Appendices}

\section{A Proof of Proposition 1}

We consider the following problem

$$
\min _{P \in D_{c}\left(P, P_{n}\right) \leq \delta} \phi^{T} E_{P}[R]
$$

or, equivalently,

$$
-\max _{P \in D_{c}\left(P, P_{n}\right) \leq \delta} E_{P}\left[(-\phi)^{T} R\right] .
$$

By checking Slater's condition and using Proposition 4 of Blanchet, Kang and Murthy (2016) we obtain the dual problem:

$$
\max _{P \in D_{c}\left(P, P_{n}\right) \leq \delta} E_{P}\left[(-\phi)^{T} R\right]=\inf _{\lambda \geq 0}\left[\lambda \delta+\frac{1}{n} \Phi_{\lambda}\left(R_{i}\right)\right]
$$


where

$$
\begin{aligned}
\Phi_{\lambda}\left(R_{i}\right) & =\sup _{u}\left\{h(u)-\lambda c\left(u, R_{i}\right)\right\} \\
& =\sup _{u}\left\{\left(-\phi^{T}\right) u-\lambda\left\|u-R_{i}\right\|_{q}^{2}\right\} \\
& =\sup _{\Delta}\left\{\left(-\phi^{T}\right)\left(\Delta+R_{i}\right)-\lambda\|\Delta\|_{q}^{2}\right\} \\
& =\sup _{\Delta}\left\{\left(-\phi^{T}\right) \Delta-\lambda\|\Delta\|_{q}^{2}\right\}-\phi^{T} R_{i} \\
& =\sup _{\Delta}\left\{\|\phi\|_{p}\|\Delta\|_{q}-\lambda\|\Delta\|_{q}^{2}\right\}-\phi^{T} R_{i} \\
& =\frac{\|\phi\|_{p}^{2}}{4 \lambda}-\phi^{T} R_{i} .
\end{aligned}
$$

Thus, (17) becomes

$$
\begin{aligned}
\max _{P \in D_{c}\left(P, P_{n}\right) \leq \delta} E_{P}\left[(-\phi)^{T} R\right] & =\inf _{\lambda \geq 0}\left\{\lambda \delta+\frac{1}{n}\left[\frac{\|\phi\|_{p}^{2}}{4 \lambda}-\phi^{T} R_{i}\right]\right\} \\
& =\inf _{\lambda \geq 0}\left\{\lambda \delta+\frac{\|\phi\|_{p}^{2}}{4 \lambda}-\phi^{T} E_{P_{n}}[R]\right\} \\
& =\sqrt{\delta}\|\phi\|_{p}-\phi^{T} E_{P_{n}}[R]
\end{aligned}
$$

or

$$
\min _{P \in D_{c}\left(P, P_{n}\right) \leq \delta} \phi^{T} E_{P}[R]=\phi^{T} E_{P_{n}}[R]-\sqrt{\delta}\|\phi\|_{p} .
$$

\section{B Proof of Proposition 2}

Introducing a slack random variable $S \equiv v$, where $v$ is a deterministic number. Then we can recast problem (7) as

$$
\begin{gathered}
\max \left\{E_{P}\left[\left(U^{T} \phi\right)^{2}\right]: E_{\pi}[c(U, R)+S]=\delta, \pi_{R}=P_{n}, \pi(S=v)=1,\right. \\
\left.E_{\pi}\left[U^{T} \phi\right]=\alpha, \pi \in \mathcal{P}\left(\mathcal{R}^{m} \times \mathcal{R}^{m} \times \mathcal{R}_{+}\right)\right\} .
\end{gathered}
$$

Define

$$
\Omega:=\{(u, r, s): c(u, r)<\infty, s \geq 0\}
$$

and let

$$
f(u, r, s)=\left[\begin{array}{c}
1_{r=R_{1}}(u, r, s) \\
\ldots \\
1_{r=R_{n}}(u, r, s) \\
\phi^{T} u \\
1_{s=v}(u, r, s) \\
c(u, r)+s
\end{array}\right] \text { and } q=\left[\begin{array}{c}
\frac{1}{n} \\
\ldots \\
\frac{1}{n} \\
\alpha \\
1 \\
\delta
\end{array}\right]
$$


Thus 19 can be written as,

$$
\max \left\{E_{\pi}\left[\left(U^{T} \phi\right)^{2}\right]: E_{\pi}[f(U, R, S)]=q, \pi \in \mathcal{P}_{\Omega}\right\} .
$$

Let $f_{0}=\mathbf{1}_{\Omega}, \tilde{f}=\left(f_{0}, f\right), \tilde{q}=(1, q), \mathcal{Q}_{\tilde{f}}:=\left\{\int \tilde{f}(x) d \mu(x): \mu \in \mathcal{M}_{\Omega}^{+}\right\}$where $\mathcal{M}_{\Omega}^{+}$ denote the set of non-negative measures on $\Omega$. If $\phi \neq 0$, then it is easy to see that $\tilde{q}$ lies in the interior of $\mathcal{Q}_{\tilde{f}}$. By Proposition 6 in Blanchet, Kang and Murthy (2016), the optimal value of problem 22 equals to that of its dual problem, i.e.,

$$
\begin{aligned}
\max \left\{E_{\pi}\left[\left(U^{T} \phi\right)^{2}\right]\right. & \left.: E_{\pi}[f(U, R, S)]=q, \pi \in \mathcal{P}_{\Omega}\right\} \\
& =\inf _{a=\left(a_{0}, \ldots, a_{n}\right) \in A}\left\{a_{0}+\frac{1}{n} \sum_{i=1}^{n} a_{i}+\alpha a_{n+1}+a_{n+2}+\delta a_{n+3}\right\},
\end{aligned}
$$

where

$$
\begin{aligned}
A:=\left\{a=\left(a_{0}, \ldots, a_{n}\right): a_{0}+\frac{1}{n} \sum_{i=1}^{n} a_{i} 1_{r=R_{i}}(u, r, s)+a_{n+1} \phi^{T} u\right. \\
\\
\left.+a_{n+2} 1_{s=v}(u, r, s)+a_{n+3}[c(u, r)+s] \geq\left(\phi^{T} u\right)^{2}, \forall(u, r, s) \in \Omega\right\} .
\end{aligned}
$$

From the definition of $A$, replacing $r=R_{i}$, we obtain that the inequality

$$
a_{0}+a_{i}+a_{n+2} \geq \sup _{(u, s) \in \Omega}\left\{\left(\phi^{T} u\right)^{2}-a_{n+3}\left[c\left(u, R_{i}\right)+s\right]-a_{n+1} \phi^{T} u\right\}
$$

holds for each $i \in\{1, \ldots, n\}$. It follows directly that

$$
\begin{aligned}
& \sup _{(u, s) \in \Omega}\left\{\left(\phi^{T} u\right)^{2}-a_{n+3}\left[c\left(u, R_{i}\right)+s\right]-a_{n+1} \phi^{T} u\right\} \\
& = \begin{cases}+\infty, & \text { if } a_{n+3}<0 \\
\sup _{u}\left\{\left(\phi^{T} u\right)^{2}-a_{n+3} c\left(u, R_{i}\right)-a_{n+1} \phi^{T} u\right\}, & \text { if } a_{n+3} \geq 0 .\end{cases}
\end{aligned}
$$

Thus, the dual problem can be expressed as

$$
\inf \left\{a_{0}+\frac{1}{n} \sum_{i=1}^{n} a_{i}+\alpha a_{n+1}+a_{n+2}+\delta a_{n+3}: a_{n+3} \geq 0, a_{0}+a_{i}+a_{n+2} \geq \sup _{u}\left\{\left(\phi^{T} u\right)^{2}-a_{n+3} c\left(u, R_{i}\right)-a_{n+1} \phi^{T} u\right\}\right\}
$$

which can be transformed into

$$
\inf _{a_{n+3} \geq 0}\left\{\frac{1}{n} \sum_{i=1}^{n} \Phi\left(R_{i}\right)+\alpha a_{n+1}+\delta a_{n+3}\right\}
$$

with

$$
\Phi\left(R_{i}\right):=\sup _{u}\left\{\left(\phi^{T} u\right)^{2}-a_{n+3} c\left(u, R_{i}\right)-a_{n+1} \phi^{T} u\right\} .
$$

Using $\lambda_{1}$ to replace $a_{n+3}$ and $\lambda_{2}$ to replace $a_{n+1}$, the dual problem becomes

$$
\inf _{\lambda_{1} \geq 0}\left\{\frac{1}{n} \sum_{i=1}^{n} \Phi\left(R_{i}\right)+\lambda_{2} \alpha+\lambda_{1} \delta\right\}
$$

where

$$
\Phi\left(R_{i}\right):=\sup _{u}\left\{\left(\phi^{T} u\right)^{2}-\lambda_{1} c\left(u, R_{i}\right)-\lambda_{2} \phi^{T} u\right\} .
$$




\section{Proof of Proposition 3}

Writing $\Delta:=u-R_{i}$, we have

$$
\begin{aligned}
\Phi\left(R_{i}\right) & =\sup _{u}\left\{\left(\phi^{T} u\right)^{2}-\lambda_{1} c\left(u, R_{i}\right)-\lambda_{2} \phi^{T} u\right\} \\
& =\sup _{u}\left\{\left(\phi^{T} u\right)^{2}-\lambda_{1}\left\|u-R_{i}\right\|_{q}^{2}-\lambda_{2} \phi^{T} u\right\} \\
& =\sup _{\Delta}\left\{\left(\phi^{T}\left(\Delta+R_{i}\right)\right)^{2}-\lambda_{1}\|\Delta\|_{q}^{2}-\lambda_{2} \phi^{T}\left(R_{i}+\Delta\right)\right\} \\
& =\sup _{\Delta}\left\{\left(\phi^{T} R_{i}\right)^{2}+\left(\phi^{T} \Delta\right)^{2}+2\left(\phi^{T} R_{i}\right)\left(\phi^{T} \Delta\right)-\lambda_{1}\|\Delta\|_{q}^{2}-\lambda_{2} \phi^{T}\left(R_{i}+\Delta\right)\right\} \\
& =\left(\phi^{T} R_{i}\right)^{2}-\lambda_{2} \phi^{T} R_{i}+\sup _{\Delta}\left\{\left(\phi^{T} \Delta\right)^{2}+2\left(\phi^{T} R_{i}\right)\left(\phi^{T} \Delta\right)-\lambda_{1}\|\Delta\|_{q}^{2}-\lambda_{2} \phi^{T} \Delta\right\} \\
& =\left(\phi^{T} R_{i}\right)^{2}-\lambda_{2} \phi^{T} R_{i}+\sup _{\Delta}\left\{\left(\|\phi\|_{p}^{2}-\lambda_{1}\right)\|\Delta\|_{q}^{2}+\left|2\left(R_{i}^{T} \phi\right)-\lambda_{2}\right|\left(\|\phi\|_{p}\|\Delta\| \|_{q}\right)\right\} .
\end{aligned}
$$

We can consider four cases: 1) $\left.\|\phi\|_{p}^{2}>\lambda_{1}, \Phi\left(R_{i}\right)=+\infty ; 2\right)\|\phi\|_{p}^{2}=\lambda_{1}, 2 R_{i}^{T} \phi \neq \lambda_{2}$, $\Phi\left(R_{i}\right)=+\infty$; 3) $\|\phi\|_{p}^{2}=\lambda_{1}, 2 R_{i}^{T} \phi=\lambda_{2}, \Phi\left(R_{i}\right)=0$; 4) $\|\phi\|_{p}^{2}<\lambda_{1}, \Phi\left(R_{i}\right)=$ $\left(\phi^{T} R_{i}\right)^{2}-\lambda_{2} \phi^{T} R_{i}+\frac{\left(2 R_{i}^{T} \phi-\lambda_{2}\right)^{2}\|\phi\|_{p}^{2}}{4\left(\lambda_{1}-\|\phi\|_{p}^{2}\right)}$.

For any of the first three cases, the value of $\frac{1}{n} \sum_{i=1}^{n} \Phi\left(R_{i}\right)$ is $+\infty$. Hence only the fourth case is non-trivial. In this case, problem (8) is transformed into

$$
\begin{aligned}
& \inf _{\lambda_{1} \geq 0, \lambda_{2}}\left[\frac{1}{n} \sum_{i=1}^{n} \Phi\left(R_{i}\right)+\lambda_{2} \alpha+\lambda_{1} \delta\right] \\
& =\inf _{\lambda_{1} \geq\|\phi\|_{p}^{2}, \lambda_{2}}\left\{\frac{1}{n} \sum_{i=1}^{n}\left[\left(\phi^{T} R_{i}\right)^{2}-\lambda_{2} \phi^{T} R_{i}+\frac{\left(2 R_{i}^{T} \phi-\lambda_{2}\right)^{2}\|\phi\|_{p}^{2}}{4\left(\lambda_{1}-\|\phi\|_{p}^{2}\right)}\right]+\lambda_{2} \alpha+\lambda_{1} \delta\right\}
\end{aligned}
$$

Define

$$
H=\frac{1}{n} \sum_{i=1}^{n}\left[\left(\phi^{T} R_{i}\right)^{2}-\lambda_{2} \phi^{T} R_{i}+\frac{\left(2 R_{i}^{T} \phi-\lambda_{2}\right)^{2}\|\phi\|_{p}^{2}}{4\left(\lambda_{1}-\|\phi\|_{p}^{2}\right)}\right]+\lambda_{2} \alpha+\lambda_{1} \delta .
$$

Taking partial derivative with respect to $\lambda_{2}$ and setting it to be 0 , we get

$$
\frac{\partial H}{\partial \lambda_{2}}=\alpha-\frac{1}{n} \sum_{i=1}^{n}\left[\phi^{T} R_{i}+\frac{\left(2 \phi^{T} R_{i}-\lambda_{2}\right)\|\phi\|_{p}^{2}}{2\left(\lambda_{1}-\|\phi\|_{p}^{2}\right)}\right]=0
$$

which implies (note that $\phi^{T} 1=1$ guarantees that $\|\phi\|_{p}^{2}>0$ )

$$
\lambda_{2}=2 \alpha-2 C \frac{\lambda_{1}}{\|\phi\|_{p}^{2}}
$$

where $C:=\alpha-\phi^{T} E_{P_{n}}[R]$. Moreover, $\lambda_{2}$ is optimal because

$$
\frac{\partial^{2} H}{\partial \lambda_{2}^{2}}=\frac{\|\phi\|_{p}^{2}}{2\left(\lambda_{1}-\|\phi\|_{p}^{2}\right)}>0
$$


We plug (31) into 30 and obtain

$$
\begin{aligned}
& \inf _{\lambda_{1} \geq 0, \lambda_{2}}\left[\frac{1}{n} \sum_{i=1}^{n} \Phi\left(R_{i}\right)+\lambda_{2} \alpha+\lambda_{1} \delta\right] \\
& =\frac{1}{n} \sum_{i=1}^{n}\left(\phi^{T} R_{i}\right)^{2}+\inf _{\lambda_{1} \geq\|\phi\|_{p}^{2}, \lambda_{2}}\left\{\frac{1}{n} \sum_{i=1}^{n}\left[-\lambda_{2} \phi^{T} R_{i}+\frac{\left(2 R_{i}^{T} \phi-\lambda_{2}\right)^{2}\|\phi\|_{p}^{2}}{4\left(\lambda_{1}-\|\phi\|_{p}^{2}\right)}\right]+\lambda_{2} \alpha+\lambda_{1} \delta\right\} \\
& =\frac{1}{n} \sum_{i=1}^{n}\left(\phi^{T} R_{i}\right)^{2}+\inf _{\lambda_{1} \geq\|\phi\|_{p}^{2}, \lambda_{2}}\left\{\frac{1}{n} \sum_{i=1}^{n}\left[\frac{\left(2 R_{i}^{T} \phi-\lambda_{2}\right)^{2}\|\phi\|_{p}^{2}}{4\left(\lambda_{1}-\|\phi\|_{p}^{2}\right)}\right]+\lambda_{2} C+\lambda_{1} \delta\right\} \\
& =\frac{1}{n} \sum_{i=1}^{n}\left(\phi^{T} R_{i}\right)^{2}+\inf _{\lambda_{1} \geq\|\phi\|_{p}^{2}}\left\{\frac{1}{n} \sum_{i=1}^{n}\left[\frac{\left(2 R_{i}^{T} \phi-2 \alpha+2 C \frac{\lambda_{1}}{\|\phi\|_{p}^{2}}\right)^{2}\|\phi\|_{p}^{2}}{4\left(\lambda_{1}-\|\phi\|_{p}^{2}\right)}\right]+\left(2 \alpha-2 C \frac{\lambda_{1}}{\|\phi\|_{p}^{2}}\right) C+\lambda_{1} \delta\right\} .
\end{aligned}
$$

Writing $\lambda_{1}=\kappa+\|\phi\|_{p}^{2}$, we have

$$
\begin{aligned}
& \inf _{\lambda_{1} \geq 0, \lambda_{2}}\left[\frac{1}{n} \sum_{i=1}^{n} \Phi\left(R_{i}\right)+\lambda_{2} \alpha+\lambda_{1} \delta\right] \\
& =\frac{1}{n} \sum_{i=1}^{n}\left(\phi^{T} R_{i}\right)^{2}+\inf _{\kappa \geq 0}\left\{\frac{1}{n} \sum_{i=1}^{n}\left[\frac{\left(R_{i}^{T} \phi-\alpha+C \frac{k+\mid \phi \|_{p}^{2}}{\|\phi\|_{p}^{2}}\right)^{2} N}{\kappa}\right]+\left(2 \alpha-2 C \frac{\kappa+\|\phi\|_{p}^{2}}{\|\phi\|_{p}^{2}}\right) C+\left(\kappa+\|\phi\|_{p}^{2}\right) \delta\right\} \\
& =\frac{1}{n} \sum_{i=1}^{n}\left(\phi^{T} R_{i}\right)^{2}+\inf _{\kappa \geq 0}\left\{\frac{C_{1}^{2}}{\|\phi\|_{p}^{2}} k+2\|\phi\|_{p}^{2}\left(\phi^{T} \bar{W}-\alpha+C\right)+\frac{1}{n} \sum_{i=1}^{n} \frac{\left(R_{i}^{T} \phi-\alpha+C\right)^{2}\|\phi\|_{p}^{2}}{\kappa}\right. \\
& \left.+2 \alpha C-2 C^{2}+\kappa\left(\delta-\frac{2 C^{2}}{\|\phi\|_{p}^{2}}\right)+\|\phi\|_{p}^{2} \delta\right\} \\
& =\frac{1}{n} \sum_{i=1}^{n}\left(\phi^{T} R_{i}\right)^{2}+2 \alpha C-2 C^{2}+\mid \phi \|_{p}^{2} \delta+\inf _{\kappa \geq 0}\left\{\frac{1}{n} \sum_{i=1}^{n} \frac{\left(R_{i}^{T} \phi-E_{P_{n}}[R] \phi\right)^{2}\|\phi\|_{p}^{2}}{\kappa}+\kappa\left(\delta-\frac{C^{2}}{\|\phi\|_{p}^{2}}\right)\right\} .
\end{aligned}
$$

If $\delta-C^{2} /\|\phi\|_{p}^{2}<0$, then the optimal value of the above problem is $-\infty$, which means that the primal problem (7) is not feasible. If $\delta-C^{2} /\|\phi\|_{p}^{2} \geq 0$, then

$$
\begin{aligned}
& \frac{1}{n} \sum_{i=1}^{n}\left(\phi^{T} R_{i}\right)^{2}+2 \alpha C-2 C^{2}+\mid \phi \|_{p}^{2} \delta+\inf _{\kappa \geq 0}\left\{\frac{1}{n} \sum_{i=1}^{n} \frac{\left(R_{i}^{T} \phi-E_{P_{n}}[R] \phi\right)^{2}\|\phi\|_{p}^{2}}{\kappa}+\kappa\left(\delta-\frac{C^{2}}{\|\phi\|_{p}^{2}}\right)\right\} \\
& =\frac{1}{n} \sum_{i=1}^{n}\left(\phi^{T} R_{i}\right)^{2}+2\left(\alpha-\phi^{T} E_{P_{n}}[R]\right) \phi^{T} E_{P_{n}}[R]+\delta\|\phi\|_{p}^{2} \\
& +2 \sqrt{\delta\|\phi\|_{p}^{2}-\left(\alpha-\phi^{T} E_{P_{n}}[R]\right)^{2}} \sqrt{\frac{1}{n} \phi^{T} \sum_{i=1}^{n}\left(R_{i}-E_{P_{n}}[R]\right)\left(R_{i}-E_{P_{n}}[R]\right)^{T} \phi} \\
& =\frac{1}{n} \sum_{i=1}^{n}\left(\phi^{T} R_{i}\right)^{2}+2\left(\alpha-\phi^{T} E_{P_{n}}[R]\right) \phi^{T} E_{P_{n}}[R]+\delta\|\phi\|_{p}^{2} \\
& +2 \sqrt{\delta\|\phi\|_{p}^{2}-\left(\alpha-\phi^{T} E_{P_{n}}[R]\right)^{2}} \sqrt{\phi^{T} \operatorname{Var}_{P_{n}}[R] \phi} .
\end{aligned}
$$


Thus, problem (7) can be written as

$$
\begin{aligned}
& \min _{\phi} \frac{1}{n} \sum_{i=1}^{n}\left(\phi^{T} R_{i}\right)^{2}+2\left(\alpha-\phi^{T} E_{P_{n}}[R]\right) \phi^{T} E_{P_{n}}[R]+\delta\|\phi\|_{p}^{2} \\
& +2 \sqrt{\delta\|\phi\|_{p}^{2}-\left(\alpha-\phi^{T} E_{P_{n}}[R]\right)^{2}} \sqrt{\phi^{T} \operatorname{Var}_{P_{n}}[R] \phi},
\end{aligned}
$$

subject to $1^{T} \phi=1$ and $\left(\alpha-\phi^{T} E_{P_{n}}[R]\right)^{2}-\delta\|\phi\|_{p}^{2} \leq 0$.

\section{Proof of Theorem 2}

Define

$$
h_{0}(R, \Sigma)=R R^{T}-\Sigma \text { and } h_{1}(R, \mu)=R-\mu .
$$

Then, by Proposition 1 of Blanchet, Kang and Murthy (2016) we have that for any given $\mu$ and $\Sigma$,

$$
\mathcal{R}_{n}(\Sigma, \mu)=\sup _{\Lambda \in \mathbb{R}^{d \times d}, \lambda \in \mathbb{R}^{d}}\left\{-E_{P_{n}}\left[\sup _{u \in \mathbb{R}^{d}}\left\{\operatorname{Tr}\left(\Lambda h_{0}(u, \Sigma)\right)+\lambda^{T} h_{1}(u, \mu)-\|u-R\|_{q}^{2}\right\}\right]\right\} .
$$

Observe that

$$
\begin{aligned}
& \sup _{u \in \mathbb{R}^{d}}\left\{\operatorname{Tr}\left(\Lambda h_{0}(u, \Sigma)\right)+\lambda^{T} h_{1}(u, \mu)-\|u-R\|_{q}^{2}\right\} \\
& =\sup _{\Delta \in \mathbb{R}^{d}}\left\{\operatorname{Tr}\left(\Lambda h_{0}(\Delta+R, \Sigma)\right)+\lambda^{T} h_{1}(\Delta+R, \mu)-\|\Delta\|_{q}^{2}\right\} \\
& =\sup _{\Delta \in \mathbb{R}^{d}}\left\{\operatorname{Tr}\left(\Lambda\left[h_{0}(\Delta+R, \Sigma)-h_{0}(R, \Sigma)\right]\right)+\lambda^{T} \Delta-\|\Delta\|_{q}^{2}\right\} \\
& +\operatorname{Tr}\left(\Lambda h_{0}(R, \Sigma)\right)+\lambda^{T} h_{1}(R, \mu) .
\end{aligned}
$$

Moreover, let us write

$$
\operatorname{Tr}\left(\Lambda\left[h_{0}(\Delta+R, \Sigma)-h_{0}(R, \Sigma)\right]\right)=\int_{0}^{1} \frac{d}{d t} \operatorname{Tr}\left(\Lambda h_{0}(R+t \Delta)\right) d t .
$$

However,

$$
\begin{aligned}
\frac{d}{d t} \operatorname{Tr}\left(\Lambda h_{0}(R+t \Delta)\right) & =2 \operatorname{Tr}\left(\Lambda(R+t \Delta) \Delta^{T}\right) \\
& =2 \operatorname{Tr}\left(\Lambda R \Delta^{T}\right)+2 t \Delta^{T} \Lambda \Delta .
\end{aligned}
$$

Furthermore,

$$
\left.E_{P_{n}}\left[\operatorname{Tr}\left(\Lambda h_{0}(R, \Sigma)\right)\right]\right|_{\Sigma=\Sigma_{n}}=0
$$

So, we deduce

$$
\begin{aligned}
& \mathcal{R}_{n}\left(\Sigma_{n}, \mu\right) \\
& =\sup _{\lambda \in \mathbb{R}^{d}}\left\{-E_{P_{n}}\left[\lambda^{T}(R-\mu)\right]+\right. \\
& \left.\sup _{\Lambda \in \mathbb{R}^{d \times d}}\left(-E_{P_{n}}\left[\sup _{\Delta}\left\{2 \operatorname{Tr}\left(\Lambda R \Delta^{T}\right)+\Delta^{T} \Lambda \Delta+\lambda^{T} \Delta-\|\Delta\|_{q}^{2}\right\}\right]\right)\right\} .
\end{aligned}
$$


Introduce the scaling $\Delta=\bar{\Delta} / n^{1 / 2}$ and $\bar{\lambda}=\lambda n^{1 / 2}$ and $\bar{\Lambda}=\Lambda n^{1 / 2}$. Then we obtain

$$
\begin{aligned}
& n \mathcal{R}_{n}\left(\Sigma_{n}, \mu_{n}\right) \\
& =\sup _{\bar{\lambda} \in \mathbb{R}^{d}}\left\{-n^{-1 / 2} \sum_{i=1}^{n} \bar{\lambda}^{T}\left(R_{i}-\mu_{n}\right)+\right. \\
& \left.\sup _{\bar{\Lambda} \in \mathbb{R}^{d \times d}}\left(-E_{P_{n}}\left[\sup _{\bar{\Delta}}\left\{2 \operatorname{Tr}\left(\bar{\Lambda} R \bar{\Delta}^{T}\right)+\bar{\Delta}^{T} \bar{\Lambda} \bar{\Delta} / n^{1 / 2}+\bar{\lambda}^{T} \bar{\Delta}-\|\bar{\Delta}\|_{q}^{2}\right\}\right]\right)\right\} .
\end{aligned}
$$

In the proof of Proposition 3 in Blanchet, Kang and Murthy (2016), under Assumption A2), a technique is introduced to show that $\bar{\Delta}$ and $\bar{\lambda}$ can be restricted to compact sets with high probability and therefore the term $\bar{\Delta}^{T} \bar{\Lambda} \bar{\Delta} / n^{1 / 2}$ is asymptotically negligible. On the other hand,

$$
\begin{aligned}
& \sup _{\Delta}\left\{2 \operatorname{Tr}\left(\bar{\Delta}^{T} \bar{\Lambda} R\right)+\bar{\Delta}^{T} \bar{\lambda}-\|\bar{\Delta}\|_{q}^{2}\right\} \\
& =\sup _{\Delta}\left\{2\|\bar{\Lambda} R+\bar{\lambda}\|_{p}\|\bar{\Delta}\|_{q}-\|\bar{\Delta}\|_{q}^{2}\right\}=\|\bar{\Lambda} R+\bar{\lambda}\|_{p}^{2} .
\end{aligned}
$$

Therefore, if

$$
n^{-1 / 2} \sum_{i=1}^{n}\left(R_{i}-\mu_{n}\right) \Rightarrow-Z
$$

for some $Z$ (to be characterized momentarily), then we conclude that

$$
\mathcal{R}_{n}\left(\Sigma_{n}, \mu_{n}\right) \Rightarrow L_{0}=\sup _{\bar{\lambda} \in \mathbb{R}^{d}}\left\{\bar{\lambda}^{T} Z-\inf _{\bar{\Lambda} \in \mathbb{R}^{d \times d}} E_{P^{*}}\left[\|\bar{\Lambda} R+\bar{\lambda}\|_{p}^{2}\right]\right\}
$$

If $p=2$ then we have

$$
E_{P^{*}}\left[\|\bar{\Lambda} R+\bar{\lambda}\|_{2}^{2}\right]=\sum_{i} E_{P^{*}}\left(\bar{\Lambda}_{i} \cdot R+\bar{\lambda}_{i}\right)^{2}
$$

So, taking derivative with respect to the $i$-th row, $\bar{\Lambda}_{i}$, of the matrix $\bar{\Lambda}, \bar{\Lambda}_{i}$, we obtain

$$
\nabla_{\bar{\Lambda}_{i}} E_{P^{*}}\left[\|\bar{\Lambda} R+\bar{\lambda}\|_{2}^{2}\right]=2 E_{P^{*}}\left(\left(R^{T} \bar{\Lambda}_{i}+\bar{\lambda}_{i}\right) R\right)=2 E_{P^{*}}\left(R^{T} \bar{\Lambda}_{i} \cdot R\right)+2 \bar{\lambda}_{i} E_{P^{*}}(R)=0 .
$$

Writing

$$
\mu_{*}=E_{P^{*}}(R) \text { and } \Sigma_{*}=E_{P^{*}}\left(R R^{T}\right),
$$

and then multiplying 34 by $\bar{\Lambda}_{i}^{T}$. we obtain

$$
\bar{\Lambda}_{i}^{T} \Sigma_{*} \bar{\Lambda}_{i}=-\bar{\lambda}_{i} \bar{\Lambda}_{i}^{T} \mu_{*} .
$$

To solve this equation take

$$
\bar{\Lambda}_{i .}=a_{i} \mu_{*}
$$

leading to

$$
a_{i} \mu_{*}^{T} \Sigma_{*} \mu_{*}=-\bar{\lambda}_{i}\left\|\mu_{*}\right\|_{2}^{2}
$$

or

$$
a_{i}=-\bar{\lambda}_{i}\left\|\mu_{*}\right\|_{2}^{2} / \mu_{*}^{T} \Sigma_{*} \mu_{*} .
$$


Therefore,

$$
\begin{aligned}
E_{P^{*}}\left(\bar{\Lambda}_{i} \cdot R+\bar{\lambda}_{i}\right)^{2} & =\bar{\Lambda}_{i}^{T} \Sigma_{*} \bar{\Lambda}_{i \cdot}+2 \bar{\lambda}_{i} \bar{\Lambda}_{i \cdot}^{T} \mu_{*}+\bar{\lambda}_{i}^{2} \\
& =\bar{\lambda}_{i}^{2}+\bar{\lambda}_{i} \bar{\Lambda}_{i \cdot}^{T} \mu_{*}=\bar{\lambda}_{i}^{2}\left(1-\left\|\mu_{*}\right\|_{2}^{4} / \mu_{*}^{T} \Sigma_{*} \mu_{*}\right)
\end{aligned}
$$

Observe that $\left\|\mu_{*}\right\|_{2}^{4} / \mu_{*}^{T} \Sigma_{*} \mu_{*}<1$ if and only if

$$
\operatorname{Tr}\left(E_{P^{*}}\left(R R^{T}\right) E_{P^{*}}(R) E_{P^{*}}\left(R^{T}\right)\right)>\operatorname{Tr}\left(E_{P^{*}}(R) E_{P^{*}}\left(R^{T}\right) E_{P^{*}}(R) E_{P^{*}}\left(R^{T}\right)\right),
$$

which in turn holds if and only if

$$
\begin{aligned}
& \operatorname{Tr}\left(E_{P^{*}}\left(R^{T}\right)\left[E_{P^{*}}\left(R R^{T}\right)-E_{P^{*}}(R) E_{P^{*}}\left(R^{T}\right)\right] E_{P^{*}}(R)\right) \\
& =\operatorname{Var}_{P^{*}}\left(E_{P^{*}}\left(R^{T}\right) R\right)>0 .
\end{aligned}
$$

It follows from A3) that $\operatorname{Var}_{P^{*}}\left(E_{P^{*}}\left(R^{T}\right) R\right)>0$. Hence,

$$
\begin{aligned}
L_{0} & =\sup _{\bar{\lambda} \in \mathbb{R}^{d}}\left\{\bar{\lambda}^{T} Z-\inf _{\bar{\Lambda} \in \mathbb{R}^{d \times d}} E_{P^{*}}\left[\|\bar{\Lambda} R+\bar{\lambda}\|_{2}^{2}\right]\right\} \\
& =\sup _{\bar{\lambda} \in \mathbb{R}^{d}}\left\{\bar{\lambda}^{T} Z-\|\bar{\lambda}\|_{2}^{2}\left(1-\left\|\mu_{*}\right\|_{2}^{4} / \mu_{*}^{T} \Sigma_{*} \mu_{*}\right)\right\} \\
& =\frac{\|Z\|_{2}^{2}}{4\left(1-\left\|\mu_{*}\right\|_{2}^{4} / \mu_{*}^{T} \Sigma_{*} \mu_{*}\right)} .
\end{aligned}
$$

It remains to identify $Z$. Observe that

$$
\begin{aligned}
\mu_{n} & =\rho 1+2\left(\Sigma_{n} \phi^{*}-\phi^{* T} \Sigma_{n} \phi^{*} 1\right) / \lambda_{1}^{*} \\
& =\rho 1+2\left(\Sigma_{*} \phi^{*}-\phi^{* T} \Sigma_{*} \phi^{*} 1\right) / \lambda_{1}^{*} \\
& +2\left(H_{n} \phi^{*}-\phi^{* T} H_{n} \phi^{*} 1\right) / \lambda_{1}^{*} \\
& =\mu_{*}+2\left(H_{n} \phi^{*}-\phi^{* T} H_{n} \phi^{*} 1\right) / \lambda_{1}^{*},
\end{aligned}
$$

where $H_{n}:=\Sigma_{n}-\Sigma_{*}$. By A1) we have

$$
\begin{aligned}
n^{-1 / 2} \sum_{i=1}^{n}\left(R_{i}-\mu_{*}\right) & \Rightarrow Z_{0} \sim N\left(0, \Upsilon_{g_{1}}\right) \\
n^{1 / 2} H_{n} & \Rightarrow Y \sim N\left(0, \Upsilon_{g_{2}}\right)
\end{aligned}
$$

Thus,

$$
\begin{aligned}
& n^{-1 / 2} \sum_{i=1}^{n} \bar{\lambda}^{T}\left(R_{i}-\mu_{*}\right)+2 n^{1 / 2} \bar{\lambda}^{T}\left(H_{n} \phi^{*}-\phi^{* T} H_{n} \phi^{*} 1\right) / \lambda_{1}^{*} \\
& \Rightarrow \bar{\lambda}^{T} Z=\bar{\lambda}^{T}\left(Z_{0}+Z_{1}\right)
\end{aligned}
$$

where

$$
Z_{1}:=2\left(Y \phi^{*}-\phi^{* T} Y \phi^{*} 1\right) / \lambda_{1}^{*}
$$




\section{References}

[1] Blanchet, J., and Kang, Y. (2017) Distributionally Robust Groupwise Regularization Estimator, arXiv:https://arxiv.org/abs/1705.04241

[2] Blanchet, J., Kang, Y., and Murthy, K. (2016) Robust Wasserstein Profile Inference and Applications to Machine Learning, arXiv:https://arxiv.org/abs/1610.05627

[3] Blanchet, J., Kang, Y., Zhang, F., and Murthy, K. (2017) Data-driven Optimal Transport Cost Selection for Distributionally Robust Optimization, arXiv:https://arxiv.org/abs/1705.07152

[4] Costa, O. and Paiva, A. (2002) Robust portfolio selection using linear-matrix inequalities, Journal of Economic Dynamics and Control, 26 , pp. 889-909.

[5] Delage, E. and Ye, Y. (2010) Data-Driven Distributionally Robust Optimization Under Moment Uncertainty with Application to Data-Driven Problems, Operations Research, 58 , pp. 595-612.

[6] Esfahani, P. and Kuhn, D. (2017) Data-Driven Distributionally Robust Optimization Using the Wasserstein Meric: Performance Guarantees and Tractable Reformulations, https://link.springer.com/article/10.1007/s10107-017-1172-1

[7] Ghaoui, L., Oks, M. and Oustry, F. (2003) Worst-case Value-at-Risk and robust portfolio optimization: A conic programming approach, Operations Research, 51, pp. 543-556.

[8] Goh, J. and Sim, M. (2010) Distributionally Robust Optimization and Its Tractable Approximations, Operations Research, 58 , pp. 595-612.

[9] Goh, J. and Sim, M. (2013) Kullback-Leibler divergence constrained distritbutionally robust optimization, Available at Optimization Online.

[10] Halldorsson, B. and Tutuncu. R. (2003) An interior-point method for a class of saddle-point problems, Journal of Optimization Theory and Applications, 116 , pp. 559-590.

[11] Hansen, L. and Sargent, T. (2008) Robustness. Princeton,N.J.:Princeton University Press.

[12] Jiang, R. and Guan, Y. (2016) Data-Drive Chance Constrained Stochastic Program, Mathematical Programming, 158, pp. 291-327.

[13] Lobo, M. and Boyd, S. (2000) The worst-case risk of a portfolio, https://web.stanford.edu/boyd/papers/pdf/risk_bnd.pdf

[14] Markowitz, H. (1952) Portfolio selection, Journal of Finance, 7, pp. 77-91.

[15] Petersen,I., James, M. and Dupuis, P. (2000) Minimax optimal control of stochastic uncertain systems with relative entropy constraints, IEEE Transactions on Automatic Control 45 (3), 398-412 
[16] Pflug, G. and Wozabal, D. (2007) Ambiguity in portfolio selection, Quantitative Finance, 7 , pp. 435-442.

[17] Villani. C. (2003) Topics in optimal transportation. Graduate Studies in Mathmatics 58, Amer. Math. Soc., Providence, RI.

[18] Wisemann, W., Kuhn, D., and Sim, M. (2014) Distributionally Robust Convex Optimization, Operations Research, 62 , pp. 1358-1376.

[19] Wozabal,D. (2012) A framework for optimization under ambiguity, Annals of Operations Research, 193 , pp. 21-47. 